\title{
Compiler Infrastructure
}

\author{
Rudi Eigenmann · Sam Midkiff
}

Received: 16 April 2013 / Accepted: 17 April 2013 / Published online: 17 July 2013

(C) Springer Science+Business Media New York 2013

This special issue presents a number of papers that discuss the design of compiler infrastructures and their use in research and education projects.

Early work described in these papers was presented at the "Cetus Users and Compiler Infrastructure Workshop," held in conjunction with the International Conference on Parallel Architectures and Compilation Techniques, PACT'2011, in Galveston Texas, on October 10, 2011. The focus on the Cetus compiler is evident in the present papers as well. An attempt was made, however, to cover other infrastructure platforms as well. To this end, papers in the special issue also discuss the LLVM, OpenUH, and Rose platforms. These special issue papers have all been reviewed according to the journal's high-quality standards for publication.

The availability of advanced compiler infrastructures is of critical importance. It enables the implementation of a new program analysis, optimization, or transformation pass in the context of a complete translation platform that can handle realistic applications and thus evaluate the new pass quantitatively. As different research projects have different needs, more than a single compiler infrastructure is needed. It is not the goal of the special issue to advertise one best infrastructure. Rather, the reader can learn about features of different platforms and identify the one that is most useful for their research plan. The included papers pursue this aim as follows:

The first article, by Bae et al., provides an overview of the Cetus compiler infrastructure. The second paper, by Yi Yang and Huiyang Zou, describes an optimizing compiler for GPGPU architectures that is built on the Cetus infrastructure. Next, Gabriel Rodríguez et al., compare two infrastructures, LLVM and Cetus, by using them in a

\footnotetext{
R. Eigenmann $(\varangle) \cdot$ S. Midkiff

Purdue University, West Lafayette, IN, USA

e-mail: eigenman@purdue.edu

S. Midkiff

e-mail: smidkiff@purdue.edu
} 
project to create a compiler that provides fault tolerance for message-passing applications. Amin Sarvestani et al. have created a tool for idiom recognition in digital signal processing applications. They describe the use of the Cetus infrastructure for that purpose. Barbara Chapman et al. present the OpenHP compiler infrastructure and its use in research and education projects. Finally, Xipeng Shen et al. describe the use of the Cetus and Rose infrastructures in the creation of an auto-tuning system that adapts compiler optimizations for GPUs based on characteristics of the program input. 